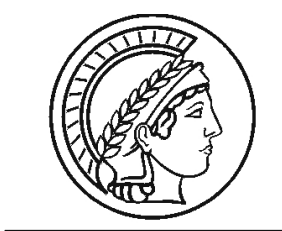

\title{
Adsorbate dynamics on iron oxide surfaces studied by scanning tunneling microscopy.
}

\author{
Sh. Shaikhutdinov ${ }^{*}$ and W. Weiss
}

Department of Inorganic Chemistry, Fritz-Haber-Institute of the MPG, Faradayweg 4-6, 14195 Berlin, Germany

${ }^{*}$ Corresponding author: e-mail shaikhutdinov@fhi-berlin.mpg.de, phone +49 3084134193

\begin{abstract}
.
Epitaxial $\mathrm{Fe}_{3} \mathrm{O}_{4}(111)$ films were studied by scanning tunneling microscopy (STM). Atomic resolution STM images exhibit a hexagonal lattice of protrusions with a $6 \AA$ periodicity which are assigned to the topmost Fe cations. In contrast to the bias polarity independent STM images obtained on the clean surface, the adsorbed species are imaged as protrusions at positive bias and as depressions at negative sample bias. They occupy top and 3 -fold hollow sites of the topmost Fe-layer, whereas no adsorbates were found on Fe vacancy sites, at step edges and on the surface domains that are $\mathrm{FeO}(111)$ in nature. The data were explained by assuming the dissociative water adsorption to occur on the regular $\mathrm{Fe}_{3} \mathrm{O}_{4}(111)$ surface, which exposes both acidic $\mathrm{Fe}$ and basic $\mathrm{O}$ sites. The diffusion of adsorbed species was studied by consecutive STM images. Both translational and rotational moving of "dimer"-species is observed.
\end{abstract}

Keywords: iron oxide, epitaxial films, scanning tunneling microscopy, adsorption 


\section{Introduction.}

Iron oxide based compounds are used as heterogeneous catalysts for various synthesis reactions [1]. Under thermodynamic equilibrium, their bulk structure and stoichiometry depend on the ambient oxygen gas pressure and temperature as described by the $\mathrm{p}\left(\mathrm{O}_{2}\right)$-T phase diagram, which can be calculated by phenomenological thermodynamics. The structure and composition at the surface cannot be predicted by simple thermodynamics, but those determine the catalytic properties as demonstrated for example in a recent investigation of the dehydrogenation of ethylbenzene to styrene over epitaxial iron oxide model catalyst films [2]. Previous studies performed on natural single crystals revealed a strong dependence of the surface structure on the preparation conditions, which was evidenced in particular by scanning tunneling microscopy (STM) (ref. 3 and ref. therein). In this paper, we present STM studies of $\mathrm{Fe}_{3} \mathrm{O}_{4}(111)$ films prepared by epitaxial growth onto a $\mathrm{Pt}(111)$ substrate. Our data show that the surface structure depends on preparation procedure, which can result in the coexistence of several different structures. The STM images of adsorbates were found to be strongly bias dependent and temporal consecutive STM images ("STM-movie") were used for studying the dynamical behavior of adsorbed species.

\section{Experimental.}

The experiments were carried out in an ultra-high-vacuum (UHV) chamber (base pressure below $10^{-10}$ mbar) equipped with a STM, a low energy electron diffraction (LEED), an Auger electron spectroscopy (AES) and other facilities [4]. The preparation and growth of the epitaxial $\mathrm{Fe}_{3} \mathrm{O}_{4}(111)$ films is described in detail in ref. [5]. Briefly, iron was evaporated onto the clean $\mathrm{Pt}(111)$ substrate, followed by oxidation in $10^{-6}$ mbar $\mathrm{O}_{2}$ at $\sim 1000 \mathrm{~K}$. This produces a $\mathrm{FeO}(111)$ monolayer film which exhibits a characteristic satellite LEED pattern. Then the oxidation temperature is decreased to $\sim 900 \mathrm{~K}$, and after repeated deposition-oxidation cycles $\mathrm{Fe}_{3} \mathrm{O}_{4}(111)$ multilayer films at least $100 \AA$ thick are obtained. A final annealing procedure was performed in $10^{-6}$ mbar $\mathrm{O}_{2}$ at $1000 \mathrm{~K}$ for $10 \mathrm{~min}$. All films exhibited characteristic LEED patterns with sharp spots and no AES contamination signals. The STM measurements were performed at room temperature in the constant current mode using electrochemically etched tungsten tips subsequently annealed in UHV by electron beam heating.

\section{Results and discussion.}

Fig. 1 shows the typical morphology of epitaxial $\mathrm{Fe}_{3} \mathrm{O}_{4}(111)$ films. Atomically flat terraces up to $1000 \AA$ in size are separated by monoatomic steps $\sim 5 \AA$ high, which are oriented along the main crystallographic directions. Shown in the inset is an atomic resolution STM image exhibiting a hexagonal lattice of protrusions with a $6 \AA$ periodicity and randomly distributed vacancy defects. These characteristic images are obtained at both positive and negative bias polarity. According to a previous LEED intensity analysis the $\mathrm{Fe}_{3} \mathrm{O}_{4}(111)$ surface is terminated by $1 / 4$ monolayer (ML) of $\mathrm{Fe}$ cations located over a close-packed oxygen layer [6], and the atomic protrusions in the STM image are assigned to these surface Fe atoms. This interpretation agrees with ab-initio spin-density functional theory calculations [7], which revealed the dominant electron density of states (DOS) near the Fermi level to be due to the Fe3d orbitals of the topmost Fe atoms. These states produce two DOS maxima located just above and below the Fermi level, that explains the bias independence of STM images obtained on the clean $\mathrm{Fe}_{3} \mathrm{O}_{4}(111)$ surface.

This regular Fe-terminated surface is produced by oxidation at temperatures above $970 \mathrm{~K}$. Several different superstructures with short-range periodicity of $3 \AA$ and $6 \AA$ are formed by preparation at lower oxidation temperatures $(\sim 870 \mathrm{~K})$, which cover about $5 \%$ of the sample surface [7]. Such a superstructure region is seen in Fig. 2. They were attributed to the "biphase ordering" phenomenon [3], where $\mathrm{FeO}(111)$-like surface domains and O-terminated $\mathrm{Fe}_{3} \mathrm{O}_{4}(111)$ domains coexist with the regular Fe-terminated $\mathrm{Fe}_{3} \mathrm{O}_{4}(111)$ surface in an ordered manner. The formation of these structures probably is induced by the slow oxidation kinetics of metallic $\mathrm{Fe}$ to $\mathrm{FeO}$ and $\mathrm{Fe}_{3} \mathrm{O}_{4}$ during preparation of the epitaxial films.

During prolonged STM measurements $\left(\sim 3\right.$ hours) we observed some atomic size features on the $\mathrm{Fe}_{3} \mathrm{O}_{4}(111)$ surface, which appearance strongly depends on the tunneling voltage in contrast to the bias independent STM images of the clean surface. To illustrate this, Fig. 2 shows two pairs of STM images of the regular $\mathrm{Fe}_{3} \mathrm{O}_{4}(111)$ surface $(a, b)$ and of the biphase superstructure region (c,d), obtained at sample voltages of +0.3 and $+0.9 \mathrm{~V}$. In addition to the characteristic atomic lattice of protrusions and vacancy defects visible at both conditions in (a) and (b), numerous bright features are observed at the higher bias voltage in (b) but not at the lower voltage in (a). Also, the indentations in (c) appear as large protrusions in (d). This effect cannot be attributed to STM tip changes since both structures could reversibly be imaged by slowly changing the bias. This is also evidenced by the fact that the protrusions with a $\sim 6 \AA$ spacing in the lower part of the images (c, d) are not affected by tunneling parameters. Because of the bias independence of STM images obtained on the clean surface, we attribute the bias dependent features to the adsorption from residual gas consisting mainly of $\mathrm{H}_{2}, \mathrm{H}_{2} \mathrm{O}, \mathrm{CO}$ and $\mathrm{CO}_{2}$.

With different tips and samples, and keeping the tunneling current in the $\sim 1 \mathrm{nA}$ range we found that the adsorbed species are seen as protrusions only at positive bias voltages higher than $\sim 0.5 \mathrm{~V}$. At negative polarity, they are not visible or are imaged as depressions. Close inspection of numerous STM images of $\mathrm{Fe}_{3} \mathrm{O}_{4}(111)$ obtained at positive bias showed different types of adsorbed species. The first type labeled (1) in Fig. 3 is imaged as a 1-2 $\AA$ high protrusion in 3-fold hollow sites of the topmost Fe layer. The type (2) occupies a hollow site too, but it exhibits a very small corrugation and appears rather as a tripod. These species can coalesce and form cloud-like patches marked $(2 *)$. Type (3) is seen as a protrusion on top sites, which sometime create "dimer" structure as indicated by arrows in Fig. 4a. At negative sample bias, the adsorbates appear as depressions, so that the surface looks clean but with a higher density of vacancy defects (Fig. 4b). In this case, the top-site species (3) are imaged similar to the vacancy defects on the clean surface; the 3 -fold hollow site species (1) are imaged as triangular depressions (1* in Fig. 4b) due to their influence on three neighboring Fe atoms.

The total coverage of adsorbed species estimated from the STM images can reach $15 \%$ of the surface Fe atom density or $\sim 0.03$ ML. The AES spectra of all samples studied showed no other elements except Fe and O. This suggests that hydrogen, water and/or hydroxyl groups, which are not detectable or indistinguishable with AES, are possible candidates for the observed species. Indeed, the 
thermal-desorption spectra obtained from the samples inspected by STM show a small water desorption signal at T 320-350K. The area of this signal corresponds to few percents of monolayer, as deduced from a calibration experiment.

The assignment of the bias dependent species to water adsorption is supported by UPS and TDS studies [11], which revealed a dissociative adsorption of water at room temperature on the regular $\mathrm{Fe}_{3} \mathrm{O}_{4}(111)$ surface. It is proposed that the resulting OH-groups get adsorbed onto iron cations and the H-species onto neighboring oxygen anions. In this context, we note that the "true" Fe vacancies seen by STM at positive bias are free of any adsorbed species, and also the terrace steps are not decorated by any adsorbates. In addition, the STM images never showed adsorbed species on the superstructure regions but only on the regular $\mathrm{Fe}_{3} \mathrm{O}_{4}(111)$ surface (compare for example the upper and lower parts on the image (b) in Fig. 2). Since the superstructure regions are assumed to consist of domains terminated by close-packed iron or oxygen layers like on the $\mathrm{FeO}(111)$ surface [7], no dissociative adsorption of water on these surface domains occurs. It seems plausible that pairs of acidic $\mathrm{Fe}$ and neighboring basic $\mathrm{O}$ sites, which exist only on the regular $\mathrm{Fe}_{3} \mathrm{O}_{4}(111)$ surface, are needed for the water dissociation.

Due to the low isosteric heat of adsorption for water on $\mathrm{Fe}_{3} \mathrm{O}_{4}(111)(\sim 50 \mathrm{~kJ} / \mathrm{mol}[11])$, we assume a high mobility of the adsorbed species at room temperature. In order to monitor this by STM, consecutive STM images of the same surface region were collected (with acquisition time being about one min per image) and then "animated" with a high speed like in a movie, which clearly visualizes the surface dynamics. Fig. 4 shows two pairs of snapshots from "STM-movies" recorded at positive (a) and negative (b) sample biases. Again, adsorbed species are seen as protrusions at positive bias and depressions at negative bias. It is very surprising to see the formation of dimer species, which move translationally and rotate around one of the "atoms" consisting of. The arrows in Fig. 4 are indicated to follow their motion. Obviously, these are not real dimer molecules since the distance between iron cations on top of which these species are located is $6 \AA$, which cannot be attributed to any bonding. Tentatively, we assign this phenomenon to an attractive behavior of the adsorbed species, which can form strings (e.g., first image in Fig. 4b) or rhombs (marked by arrow in the last image in Fig. 4a).

Another STM movies (not presented here) of the surfaces like imaged in Fig. 4 reveal that species (2) can stick and leave the islands $(2 *)$ and therefore change the island shape. All types of adsorbed species diffuse on the surface by hopping into the nearest empty adsorption sites, i.e., from top into the top or from 3-fold into the 3-fold sites. We never observed any moving of the Fe vacancies which could be detected at positive sample bias. This implies no structural changes of the iron oxide surfaces to occur at room temperature, although this could be concluded from the STM movies obtained at negative sample bias (see Fig. 4b) if one assigns all depressions to vacancy defects. Therefore, a careful analysis of STM images obtained at various tunneling parameters must be done before making some conclusions about the dynamical behavior of these surfaces.

It should be also mentioned that the STM images of the $\alpha-\mathrm{Fe}_{2} \mathrm{O}_{3}(0001)$ hematite surface reveal bias dependent adsorbate-like features, too. However, the preliminary results favor no any diffusion of adsorbed species on hematite surface under conditions used.

\section{Conclusions.}

Our data show that a visualization of adsorbate species by STM depends crucially on the tunneling conditions. Adsorbed species on the $\mathrm{Fe}_{3} \mathrm{O}_{4}(111)$ surface are imaged as protrusions at positive bias and as depressions at negative sample bias. They occupy top and 3fold hollow sites of the topmost Fe layer and are attributed eventually to water adsorption from residual gas. The bias-dependent species were observed only on the surfaces exposing both $\mathrm{Fe}$ and $\mathrm{O}$ atoms in top layer. No adsorbates are found on the Fe vacancy sites, at step edges and on the $\mathrm{FeO}(111)$-like surface domains. We propose that pairs of acidic $\mathrm{Fe}$ and neighboring basic $\mathrm{O}$ sites, which are present on the regular $\mathrm{Fe}_{3} \mathrm{O}_{4}(111)$ surface, are needed for the dissociative water adsorption.

STM movies clearly indicates a surface diffusion of adsorbates on the $\mathrm{Fe}_{3} \mathrm{O}_{4}(111)$ surface. Hopping diffusion as well as translational and rotational moving of "dimer"-species are observed. Our results indicate that STM in movie mode can be very useful for studying the adsorbate surface dynamics on oxide systems, as recently demonstrated for the $\mathrm{TiO}_{2}(110)$ surface [12].

\section{References.}

[1] J.W. Geus, Appl. Catal. 25 (1986), 313.

[2] W. Weiss, D. Zscherpel and R. Schloegl, Cat. Lett. 52 (1998) 215.

[3] N.G. Condon, F.M. Leibsle, T.Parker, A.R. Lennie, D.J. Vaughan, G.Thornton, Phys. Rev. B, 55 (1997), 15885.

[4] W. Weiss, M. Ritter, D. Zscherpel, M. Swoboda, R. Schloegl, J. Vac. Sci. \& Technol. A16 (1998), 21.

[5] W. Weiss and M. Ritter, Phys. Rev. B59 (1999), 5201.

[6] M. Ritter and W. Weiss, Surf. Sci., 432 (1999) 81.

[7] Sh. Shaikhutdinov, M.Ritter, X.-G. Wang, H.Over, W. Weiss, accepted to Phys.Rev.B

[8] X.-G. Wang, W. Weiss, Sh. Shaikhutdinov, M. Ritter, M. Petersen, F. Wagner,

R. Schloegl and M. Scheffler Phys. Rev. Lett. 81 (1998), 1038.

[9] Sh. Shaikhutdinov and W. Weiss, Surf. Sci. Lett. 432 (1999) L627.

[10] R.W.G. Wyckof, Crystal structures, 2-nd ed., Vols. 1-3 (Krieger, Malabar, 1982).

[11] Sh. Shaikhutdinov, Y. Joseph, C. Kuhrs, W. Ranke, W. Weiss, to be published in Faraday Discussion 114 (1999).

[12] Y.Iwasawa, Surf.Sci., 402-404 (1998) 8. 


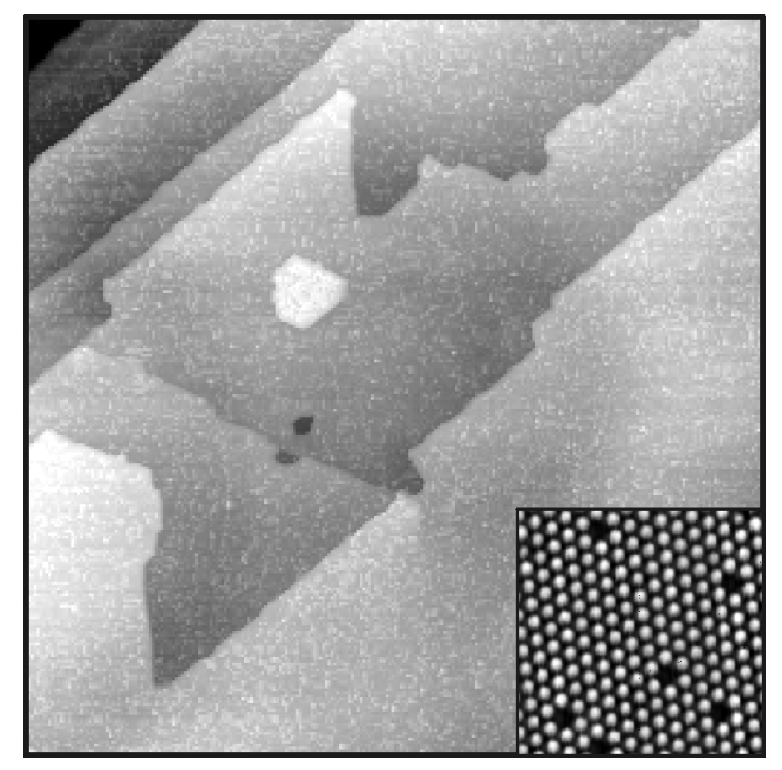

Fig. 1. Large-scale $3000 x 3000 \AA^{2}$ STM image of an epitaxial $\mathrm{Fe}_{3} \mathrm{O}_{4}(111)$ film. Shown in the inset is an $80 \mathrm{x} 80 \AA^{2}$ image of the regular $\mathrm{Fe}_{3} \mathrm{O}_{4}(111)$ surface, which exhibits a hexagonal lattice of protrusions with a $6 \AA$ periodicity and vacancy defects. Tunneling parameters: $\mathrm{V}_{\mathrm{s}}$ $=1.4 \mathrm{~V}, \mathrm{~J}=0.9 \mathrm{nA}$ and $\mathrm{V}_{\mathrm{s}}=-0.9 \mathrm{~V}, \mathrm{~J}=0.5 \mathrm{nA}$ (inset).
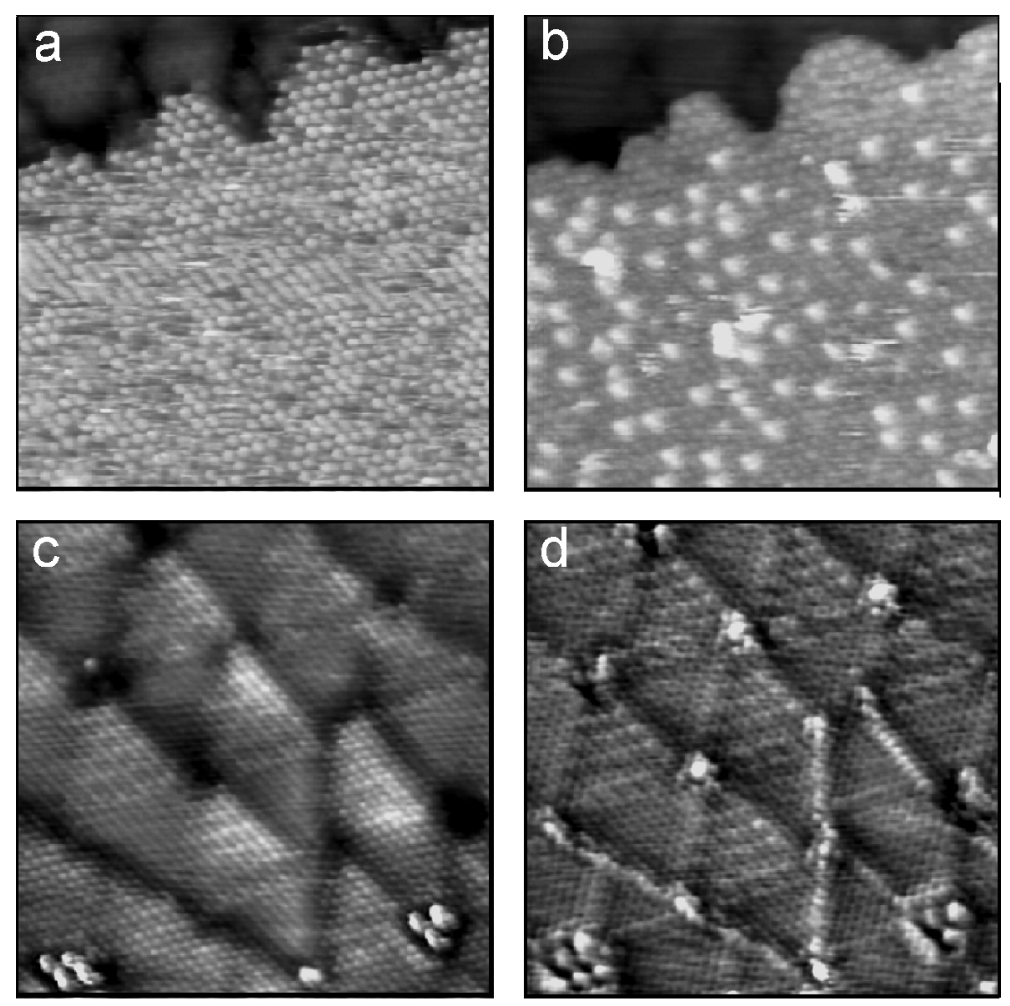

Fig. 2. Two pairs of STM images obtained on the regular $\mathrm{Fe}_{3} \mathrm{O}_{4}(111)$ surface $(\mathrm{a}, \mathrm{b})$ and on a superstructure region $(\mathrm{c}, \mathrm{d})$ taken at +0.3 $(\mathrm{a}, \mathrm{c})$ and $+0.9 \mathrm{~V}(\mathrm{~b}, \mathrm{~d})$ sample bias voltages. In addition to the characteristic atomic lattice of protrusions and vacancy defects visible at both conditions, numerous bright spots are observed at $+0.9 \mathrm{~V}$ (b) but not at $0.3 \mathrm{~V}$ (a). Also, the indentations in the image (c) appear as a protrusion in $(\mathrm{d})$. 


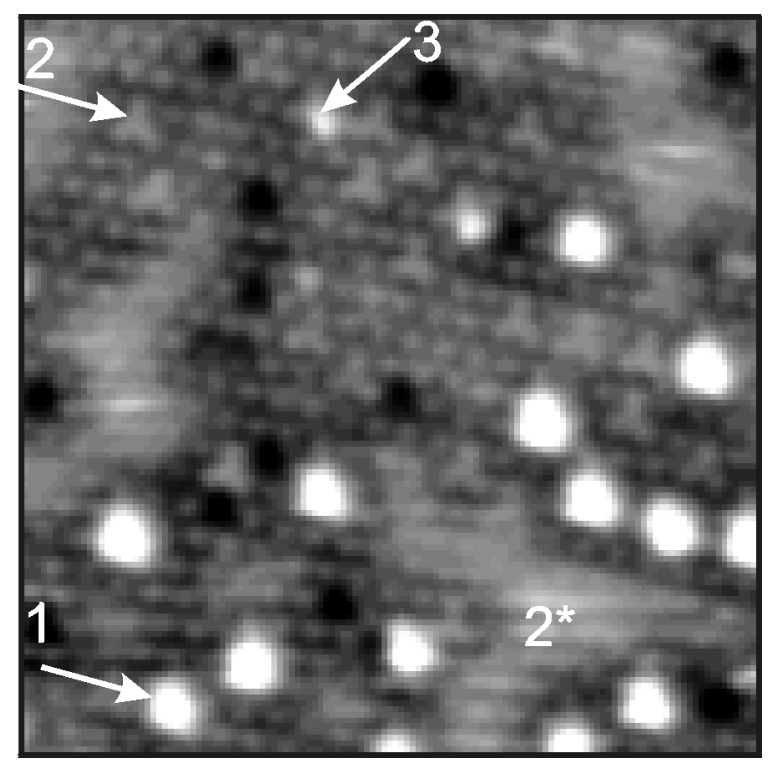

Fig. 3. STM image illustrating different types of adsorbed species on the $\mathrm{Fe}_{3} \mathrm{O}_{4}(111)$ surface, observed after few hours of measurements (see explanation in the text ). $\mathrm{V}_{\mathrm{s}}=1.25 \mathrm{~V}, \mathrm{~J}=0.9 \mathrm{nA}$. 

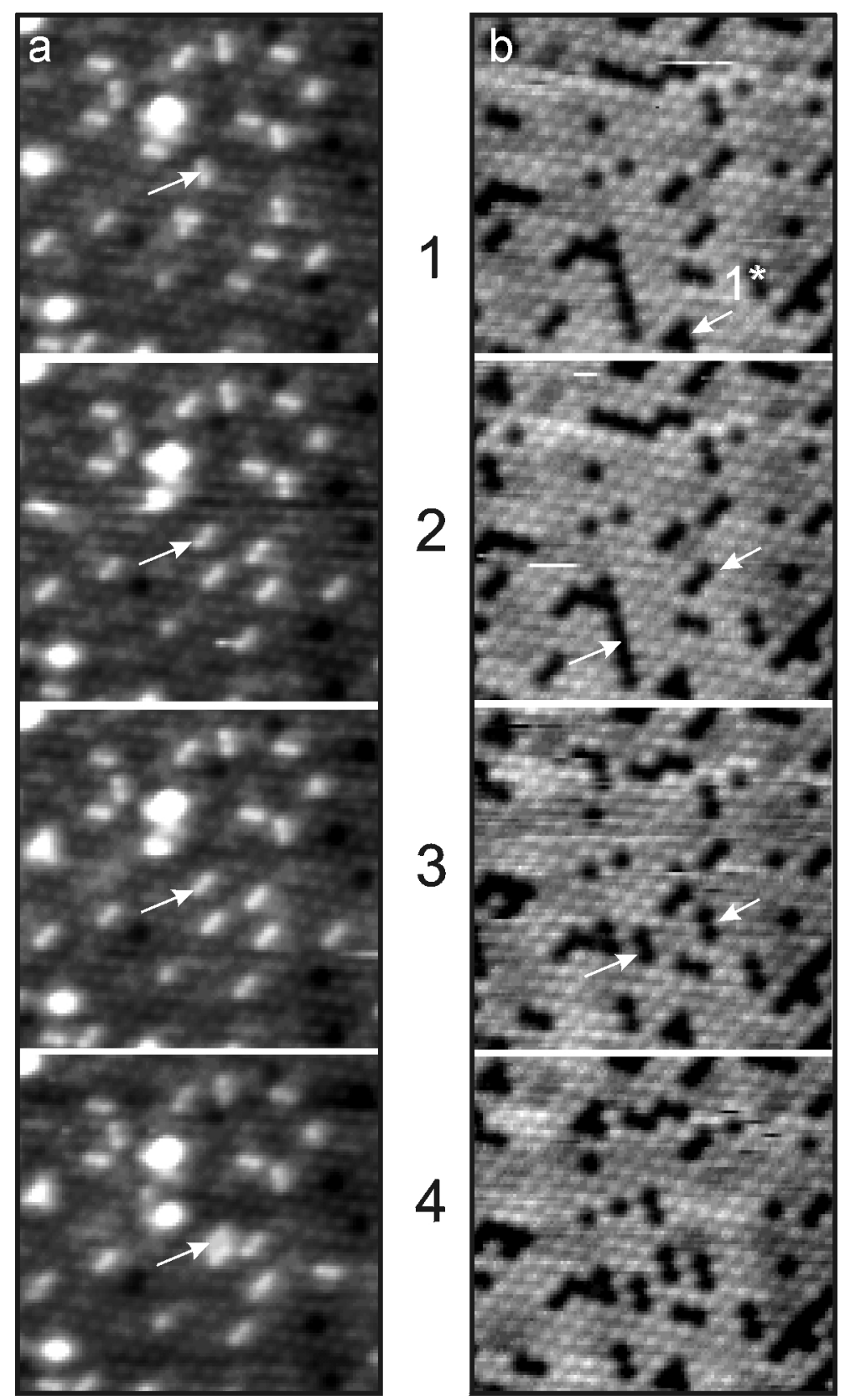

Fig. 4. Two pairs of image frames from an STM-movie recorded at positive (a) and negative (b) sample bias, number 1-4 indicates the temporal sequence. Adsorbed species are seen as protrusions at positive bias (a) and as depressions at negative bias (b). In (a) the dimer species indicated by the arrows rotates around one of its two "atoms"(frames 1-2), then it moves translationally (3-4). In (b) the arrows indicate that the dimer on the left moves, the right one rotates. $\mathrm{V}_{\mathrm{s}}=+1.3 \mathrm{~V}$ (a) and $-1.0 \mathrm{~V}$ (b), $\mathrm{J} \sim 1 \mathrm{nA}$. 